Editorial 


\section{El cuerpo: aproximaciones heterodoxas}

\section{Carlos Olivier Toledo}

Universidad Autónoma de México, México

oliviertc@iztacala.unam.mx

\author{
Alejandra Olvera Rabadán \\ Universidad Michoacana de San Nicolás de \\ Hidalgo, México \\ alejandra.olvera@umich.mx

\section{Juan Fernando Cáceres \\ Universidad Distrital Francisco José de Caldas, Colombia \\ cijuanf@correo.udistrital.edu.co}

\section{Ronald Céspedes} \\ Universidad Mayor Real y \\ Pontificia de San francisco Xavier \\ de Chuquisaca, Bolivia \\ ronald.cespedesb@gmail.com \\ Editorialistas invitados
}

Cómo citar este artículo: Olivier Toledo, Carlos: Olvera Rabadán, Alejandra; Cáceres, Juan Fernando; Céspedes, Ronald (2021). El cuerpo: aproximaciones heterodoxas. Estudios Artísticos: revista de investigación creadora, 7(10) pp. 10-14. DOI: https://doi.org/10.14483/25009311.17519

$<$

Aliento: performance vocal. (2019). Intérprete: Sirley Martínez. Dirección: Juan Fernando Cáceres.Fotografía: Gustavo García
Vivimos en la era del cuerpo. No sabemos hasta cuándo el mundo académico mantendrá el interés, sin embargo, nadie podrá dudar que en la actualidad el cuerpo es parte, ya sea como accesorio o como objeto principal, de la mayoría de los estudios académicos. Desde el mundo de las artes hasta el de las ciencias, se ha reconocido que sin él no es posible comprender del todo al hombre vivo, al hombre de carne y hueso... al hombre total. En efecto, "puesto que nuestros cuerpos -afirmó Bryan Turner en El Cuerpo y la sociedad. Exploraciones en teoría social en la década de los ochenta- son parte de la naturaleza, el trabajo debe comprender el trabajo sobre nuestros cuerpos, los que son transformados en las prácticas culturales y políticas".

Muy probablemente, el despunte de los estudios corporales puede ser hallado a partir de la segunda mitad del siglo XX, sin embargo, fue a partir de la última década que tales estudios obtuvieron una especie de carta de naturalización e independencia, a grado tal que en las universidades tanto en Estados Unidos, como Europa y América Latina se comenzaron a llevar a cabo seminarios, talleres, cursos y congresos en donde el centro de discusión era el cuerpo y la corporalidad. También se produjo toda una serie de bienes culturales dedicados a la comprensión y reflexión acerca del cuerpo, desde una amplia y variada producción bibliográfica hasta la creación de revistas desde diversos enfoques y áreas de conocimiento. ${ }^{1}$

1 En un esfuerzo de gran envergadura, la colombiana Claudia Mallarino ha realizado un rastreo de las principales producciones bibliográficas en torno al cuerpo en Latinoamérica. Cf. Claudia Mallarino, Escrituras del cuerpo y sobre el cuerpo en Colombia y América Latina a partir de la última década del siglo XX. Apuntes para una genealogía, documento inédito. 
El hecho es que los estudios corporales entraron por la puerta principal en el siglo XXI y fue por ello que se creyó necesario reflexionar sobre estos $y$, simultáneamente, tener una perspectiva de horizonte que permitiera comprender el rumbo que tales estudios estaban tomando. ¿Cuáles eran los intereses que los académicos y estudiantes estaban teniendo con respecto al cuerpo? Para obtener la respuesta era necesario convocar a estudiosos del tema para conocer las investigaciones del día, aquellas, diríamos según una frase mexicana, «recién salidas del comal».

Fue por lo anterior que en 2005 se convocó desde la UNAM al 1er Congreso Internacional "El cuerpo en el siglo XXI", un evento interesante porque fue el primer contacto que se tuvo con algunos estudiosos del cuerpo. Sin embargo, a pesar de lo interesante que pudo haber sido, ese primer evento no ofrecía una diferencia fundamental con respecto a otro tipo de eventos llevados a cabo en México, debido a que el rumbo que tomó consistió en aglutinar estudios pertenecientes al orden de la clínica. Como ya se sabe, uno de los temas que tuvo mayor despunte durante el último tercio del siglo XX fue el de los padecimientos llamados psicosomáticos, además de la relación entre las prácticas meditativas y el cuerpo como centro del escenario de la cura. Aunque hubo mesas que discutieron sobre el biopolítica y la construcción social del cuerpo, en general, todo parecía indicar que el llamado a este evento aún estaría guiado por el campo de la salud.

Nos falta espacio para detenernos en ciertos procesos que permitirían comprender la traslación producida entre el primer y segundo congreso. Basta decir que entre uno y otro median seis años, del 2005 al 2011. En México, un periodo complejo marcado por el ascenso del Partido Acción Nacional a la presidencia y también por el aumento de la narcoviolencia. Cualquiera que tenga su residencia en este país sabrá que los índices de violencia, de asesinatos, tortura y desapariciones forzadas han tenido un crecimiento paulatino, apareado con la corrupción, siempre presente, del gobierno de turno. Sin embargo, aunque hoy en día México está en la mira del mundo, en general, Latinoamérica no está exenta de tal condición: desde Colombia que sigue padeciendo las luchas entre diversos carteles, hasta Perú o Guatemala que viven en carne propia la pobreza extrema. Ante esto, pocos intelectuales habrían podido avizorar que los estudios corporales tomarían un rumbo inédito hasta entonces.

En el 2011 se convocó al 2do Congreso Internacional "El cuerpo en el Siglo XXI", al que le incluimos el subtítulo de: "Aproximaciones Heterodoxas desde Latinoamérica". No podía ser de otro modo, ya que la sede del evento sería en el Centro de Investigaciones sobre América Latina y el Caribe (CIALC) al interior de la Universidad Nacional Autónoma de México (UNAM). La línea del evento era más que evidente: nos interesaba convocar a aquellos estudiosos que estuviesen trabajando tópicos y enfoques inéditos. No es que el tema del cuerpo y la psicosomática no importasen, simplemente queríamos creer que los estudios corporales no podian ser reductibles al campo clínico. Más aún, estábamos convencidos que para generar algún cambio social de fondo las reflexiones sobre la psicosomática no eran suficientes, había que trasladarse al mundo social, al mundo que consideramos como la carne y sangre de la vida.

La convocatoria no dejó de sorprendernos. Estudiosos de Chile, Brasil, Estados Unidos (San Francisco, New Jersey), Canadá, de la Universidad de Harvard y, principalmente, de Colombia, además de las instituciones establecidas al interior de la república mexicana (Guanajuato y Morelos, además de las universidades locales) acudieron al llamado y abordaron temas que revelaban un primer panorama del rumbo que los estudios corporales estaban teniendo: transexualidad, homosexualidad; identidad en cuerpos africanos radicados en México; cuerpo, exclusión e inmigración; el cuerpo en las minorías religiosas; cuerpo, estigma y violencia; cuerpo social y espacios habitacionales; el cuerpo femenino en el circo; el abuso del cuerpo por el control territorial; el cuerpo como resistencia hegemónica en la lucha del movimiento estudiantil en Chile; cuerpo, glosalia y don de sanación en grupos pentecostales y el cuerpo en la sociedad red son solo algunos de los temas que confluyeron en este evento. Para los organizadores fue evidente la necesidad de seguir convocando y comprendiendo el fenómeno que estaba frente a nosotros. Por si la sorpresa anterior fuera poca, al siguiente evento se sumó una serie de tópicos que rebasaron nuestras propias expectativas y 
pensamientos sobre lo que se podía lograr con los estudios corporales. De modo paulatino, los eventos subsecuentes fueron tomando una especie de «rostro de denuncia»: El uso del cuerpo muerto como mensaje en las guerras internas de Perú; México, supervivencia en un contexto de guerra al narcotráfico. Paisaje fracturado y geografías corporales del castigo; La burla y lo abyecto: deconstrucción, montaje y exhibición del cuerpo muerto en México; Cholos y Maras. Dos subculturas escritas en el cuerpo; Violencia y cristianismo; Exhibición del cuerpo violentado en producciones cinematográficas con intención de denuncia: el caso de los feminicidios en Ciudad Juárez; Descivilización del cuerpo pandillero y su reclusión para la muerte: mecanismos de sostenimiento de desigualdad, poder y violencia social. Esos títulos reflejan con mayor claridad el rumbo inédito que el evento comenzó a tomar hasta llegar a la quinta versión del congreso, con la presentación del libro de Antonio Sustaita, el cual está contenido en un marco teórico heterodoxo y de una temática más que actual en nuestro país: el descabezamiento de los cuerpos.

Hay que confesar que en este momento no somos capaces de comprender el sentido de las temáticas que sintéticamente hemos presentado; lo que es un hecho es que los estudios corporales cada vez más están naciendo de la necesidad de ponerle texto a lo indecible, a lo doloroso y horroroso, a aquello que en otros tiempos pertenecía al campo de lo amarillista y de lo que toda sociedad se negaba a ver. ¿Será por ello mismo que los investigadores han tenido que tocar los «horrores del cuerpo» $y$, en lo asqueroso, haber descubierto un saber? ¿Será que en la «repugnancia» de un cuerpo ensangrentado, descuartizado o torturado el investigador des-cubrió algo en torno a sí, al nosotros y no solo a los otros?

Nos encontramos ante un hecho innegable. Por un lado, vivimos en un tiempo de nuevos mecanismos de sufrimiento, tortura y aniquilamiento y parece ser que el cuerpo ha vuelto a ser el escenario principal, ya que no solo estamos ante el descabezamiento de cuerpos. ¿Qué pretenden los carteles, por ejemplo, al separar las extremidades del cuerpo? Con la cabeza se pierde el rostro, pero con las manos y dientes, la identidad. Será que han descubierto nuevas formas de invisibilizar al otro, borrar su cuerpo a grado tal que ni los descendientes sepan de él. En la época medieval, la lección era trozar al cuerpo para colocar sus partes en cada extremo geográfico. El mensaje era: ver los pedazos de un cuerpo rebelde. Pero con las tácticas actuales se busca que el cuerpo sea irreconocible y, para ello, se ha desarrollado una estrategia casi artística que busca la invisibilidad: el mensaje ya no es ver lo trozado pero reconocible del cuerpo, ahora se trata de una carne sin identidad, sin subjetividad. Esto es aún peor, porque el cuerpo rebelde puede ser reconocido y enterrado, aún conserva una historia y un futuro, una esperanza que interpela al bienestar en otro mundo. Pero un cuerpo trozado, sin identidad, no tiene ni historia ni futuro y, por lo tanto, el dolor no se va con él, sino que se queda con el descendiente porque es peor tener un cuerpo desaparecido, así a secas, que uno muerto, trozado, pero con historia y futuro, con la esperanza de una vida mejor en un mundo más allá.

Por otro lado, el interés de los investigadores por el cuerpo violentado, destazado, desposeído, aniquilado o escamoteado puede reflejar su interés por responder a lo que hasta ahora se ha querido negar: la nueva dinámica que vive la sociedad en torno a su propia decadencia. Se trata de reflexionar sobre lo atroz, sobre el lado oscuro de una sociedad que se juega entre el horror del cuerpo violentado y la fascinación por el sufrimiento producido. Pero también, de alimentar la seducción al indagar en aquello que no se puede, no se debe ver. ¿Será que en estos tiempos necesitamos de lo indecible, invisible e intocable para encontrar el sentido de una existencia cada vez más dura e imponente?

El cuerpo no es más un objeto de estudio que está a distancia, como si este fuera un objeto inerte. Para dicha o desdicha, los estudios corporales nos atraviesan; juegan con nuestra vida psíquica, seducen aunque aterroricen. Con este giro corporal es innegable que nosotros estamos puestos en juego hasta la médula de nuestros huesos. El cuerpo maltratado o aniquilado está como dato en el foco de nuestra conciencia social, en la memoria de una sociedad que sufre y simultáneamente lastima. Está, finalmente, como referencia de una historia que ocasionalmente preferiríamos no poseer. 
sociedad y cuerpo humano; los imaginarios colectivos que se tienen sobre los cuerpos; la mirada

Con todas estas inquietudes, y otras que se han venido sumando a través de los años, se realizó en el año 2019 en la Facultad de Artes ASAB de la Universidad Distrital Francisco José de Caldas (Bogotá, Colombia), la novena versión de este congreso, siendo esta la primera vez que el evento se realizaba por fuera de México. La universidad bogotana recibió a más de 120 ponentes de diferentes lugares de América, quienes durante tres días y junto a los asistentes, debatieron diferentes aproximaciones que sobre el cuerpo y las corporeidades tienen los investigadores y creadores del continente.

El evento sirvió para consolidar aún más el trabajo que en red se viene adelantando, a través de la Línea de Investigación Excrituras Artísticas y Goces Transdiscursivos de la Maestría en Estudios Artísticos de la Universidad Distrital, con la UNAM y la Universidad Michoacana de San Nicolás de Hidalgo, ambas instituciones mexicanas, y la Universidad Mayor y Pontificia de San Francisco Xavier Chuquisaca, con sede en Bolivia. De la misma manera, sirvió para evidenciar la transversalidad que tiene el estudio de las corporeidades en la Facultad, al ser abordado desde diferentes perspectivas tanto dentro de los currículos de pregrado y posgrado, como por fuera de ellos.

Entre los ejes trabajados estuvieron: las concepciones del cuerpo en el devenir histórico; las diversidades sexuales y la experiencia corporal; el arte, la estética y la corporalidad; las políticas del cuerpo instituido; las representaciones del cuerpo en grupos juveniles; las relaciones con la reclusión y la exclusión; los abordajes desde la teoría social y la psicología; las implicaciones entre tecnología, desde la religión y la espiritualidad; las condiciones propias y adquiridas de los cuerpos en medio de la migración y el desplazamiento forzado; así como también asuntos como la virtualidad, la discapacidad, la etnicidad, la identidad y la muerte, entre otros.

De las numerosas ponencias presentadas, se hizo una selección para que las más representativas fueran publicadas en Estudios Artísticos: revista de investigación creadora y en un libro compilatorio, con el apoyo del Doctorado en Estudios Artísticos de la Universidad Distrital. Por ello, en el número anterior a esta publicación se incluyeron los artículos Símbolo de legalidad: interpretaciones artísticas a partir del cuerpo religioso de Camila Alexandra Ruiz Cruz; Fantasmas, espectros, sirenas y otros zumbidos. Otros cuerpos recorren el mundo de Jaidy Astrid Díaz Barrios; Del cuerpo natural al cuerpo cosificado: Una revisión de los modos de interacción médico-paciente en el parto de Rubén Darío Noriega De La Rosa y Menstruación: de los imaginarios a la imaginación de Stephanny Parra Ordóñez de Valdés. Igualmente, en el presente número se incluyen otros cuatro artículos que derivan de ponencias realizadas durante el IX Congreso, a saber: Al compás sensual que marca tu cintura. Otras formas de apropiación desde la experiencia de vida de Oswaldo Marchionda Vargas; Habitar el cuerpo abyecto: realización escénica a partir de un laboratorio escénico de Amanda Méndez Ramírez; Muerte y resurrección de lo negro en el museo de Arley Buitrago Landázuri y Voguing, un grito retorcido contra la opresión. Soy todo y nada, sobre la danza de cuerpos desviantes de Lucelina Nunes Barbosa. 